\title{
$h$-polynomials via reduced forms
}

\author{
Karola Mészáros* \\ Department of Mathematics \\ Cornell University \\ Ithaca, NY 14853 \\ karola@math. cornell.edu
}

Submitted: Apr 7, 2015; Accepted: Oct 12, 2015; Published: Oct 30, 2015

Mathematics Subject Classifications: 05E15, 05E45, 52B20

\begin{abstract}
The flow polytope $\mathcal{F}_{\widetilde{G}}$ is the set of nonnegative unit flows on the graph $\widetilde{G}$. The subdivision algebra of flow polytopes prescribes a way to dissect a flow polytope $\mathcal{F}_{\widetilde{G}}$ into simplices. Such a dissection is encoded by the terms of the so called reduced form of the monomial $\prod_{(i, j) \in E(G)} x_{i j}$. We prove that we can use the subdivision algebra of flow polytopes to construct not only dissections, but also regular flag triangulations of flow polytopes. We prove that reduced forms in the subdivision algebra are generalizations of $h$-polynomials of the triangulations of flow polytopes. We deduce several corollaries of the above results, most notably proving certain cases of a conjecture of Kirillov about the nonnegativity of reduced forms in the noncommutative quasi-classical Yang-Baxter algebra.
\end{abstract}

Keywords: flow polytope; subdivision algebra; reduced form; triangulation; $h$ polynomial; nonnegativity; quasi-classical Yang-Baxter algebra.

\section{Introduction}

Nonnegativity properties abound in mathematics, and whenever one arises, the most satisfying explanation of integer nonnegativity is to demonstrate what a certain nonnegative quantity counts. The present paper is written in this spirit and explains nonnegativity properties of polynomials using geometric interpretations for their coefficients.

We study dissections of flow polytopes via the subdivision algebra, and show that we can construct regular flag triangulations of flow polytopes using the subdivision algebra. This in turn empowers us to prove several interesting corollaries, one in particular partially

*The author was partially supported by a National Science Foundation Postdoctoral Research Fellowship (DMS 1103933) and a National Science Foundation Grant (DMS 1501059). 
proving a nonnegativity conjecture of Kirillov [6, Conjecture 2] of certain polynomials called reduced forms in the quasi-classical Yang-Baxter algebra.

With our geometric methods we can study reduced forms in the subdivision algebra and the quasi-classical Yang-Baxter algebra. The latter algebra was introduced by A.N. Kirillov $[5,6,7]$ with Schubert calculus in mind and it is closely related to the FominKirillov algebra [4]. The subdivision algebra has been considered by the present author under this name, as its relations encode ways to subdivide root and flow polytopes $[9,8$, 11]. It has also been considered by Kirillov $[5,6,7]$ since it is the abelianization of the quasi-classical Yang-Baxter algebra (and this is how he refers to it). The polynomials of interest in this paper arise as reduced forms in the above algebras; the reduced form of a monomial in an algebra is obtained via substitution rules dictated by the relations of the algebra.

The essence of the subdivision algebra is that the reduced form of a monomial in it can naturally be seen as a dissection of a flow polytope corresponding to the monomial into simplices. We show that among these dissections that the reduced form can encode are also unimodular, regular and flag triangulations of flow polytopes, see Theorem 10. Using the connection of reduced forms to dissections we show that reduced forms are multivariate generalizations of $h$-polynomials, see Theorem 16 . Recall that the $h$-polynomial of a simplicial complex is a way of encoding the number of faces of each dimension. We prove that if we set certain variables of reduced forms to 1 in the subdivision algebra we obtain the (shifted) $h$-polynomials of regular triangulations of flow polytopes. This result opens a new avenue for understanding reduced forms of monomials in the subdivision and related algebras. We prove nonnegativity results in the subdivision algebra as a consequence of the specialized reduced form equaling the shifted $h$-polynomial, see Theorem 21 . As a corollary we establish a special case of Conjecture 2 of Kirillov appearing in [6] about the nonnegativity of reduced forms in the quasi-classical Yang-Baxter algebra, see Theorem 23. We also express specialized reduced forms in terms of Ehrhart series of flow polytopes, which in turn can be seen in terms of Kostant partition functions, see Theorem 18 and Lemma 24.

Our methods in this paper are largely geometric. In [10] we study reduced forms from the point of view of the structure of reduction trees, leaving the geometry behind.

The paper is organized as follows. In Section 2 we define flow polytopes. Next we explain how to subdivide flow polytopes and how we can encode the subdivisions with a reduction tree. Then we define the subdivision algebra and show that the reduced form can be read off from the leaves of the reduction tree. In Section 3 we show that we can use the subdivision algebra and arrive not only at a dissection of a flow polytope, but also to a triangulation of the flow polytope, in the sense of a simplicial complex. To do this we use a particular reduction order $\sigma$. The triangulation we obtain is regular and flag. In Section 4 we prove that the reduced form of a monomial in the subdivision algebra specialized at certain variables is equal to the shifted $h$-polynomial of the aforementioned triangulation of the flow polytope of the associated to the monomial. In Section 5 we describe the full set of leaves of the reduction tree in order $\sigma$, or equivalently, the monomials in the reduced form of a monomial. In Section 6 we use the regularity of the triangulation we 
constructed to prove and interpret the nonnegativity of the coefficients of the reduced form of a monomial in the subdivision algebra as well as a special case of Conjecture 2 of Kirillov appearing in [6]. In Section 7 we relate reduced forms to Ehrhart series of flow polytopes, and thus obtain a generalization of [6, Theorem 3.10]. We also relate reduced forms to Kostant partition functions.

\section{Definitions and more of the story}

\subsection{Flow polytopes and their subdivisions}

Given a loopless graph $G$ on the vertex set $[n]$, let in $(e)$ denote the smallest (initial) vertex of edge $e$ and $\operatorname{fin}(e)$ the biggest (final) vertex of edge $e$. Let $E(G)=\left\{\left\{e_{1}, \ldots, e_{l}\right\}\right\}$ be the multiset of edges of $G$. We correspond variables $x_{e_{i}}, i \in[l]$, to the edges of $G$, of which we think as flows. The flow polytope $\mathcal{F}_{G}$ is naturally embedded into $\mathbb{R}^{l}$, where $x_{e_{i}}, i \in[l]$, are thought of as the coordinates. $\mathcal{F}_{G}$ is defined by

$$
\begin{gathered}
x_{e_{i}} \geqslant 0, i \in[l] \\
1=\sum_{e \in E(G), \operatorname{in}(e)=1} x_{e}=\sum_{e \in E(G), \operatorname{fin}(e)=n} x_{e}
\end{gathered}
$$

and for $2 \leqslant i \leqslant n$

$$
\sum_{e \in E(G), \operatorname{fin}(e)=i} x_{e}=\sum_{e \in E(G), \operatorname{in}(e)=i} x_{e} .
$$

The flow polytope $\mathcal{F}_{K_{n+1}}$, where $K_{n+1}$ is the complete graph on $n+1$ vertices, can be thought of as the Chan-Robbins-Yuen polytope [2], and has received a lot of attention, since its volume is equal to $\prod_{k=0}^{n-2} \operatorname{Cat}(k)$, where $\operatorname{Cat}(k)=\frac{1}{k+1}\left(\begin{array}{c}2 k \\ k\end{array}\right)$ is the $k$ th Catalan number. There is no combinatorial proof of the aforementioned result; Zeilberger [19] provided an analytical proof. For more of the story see [13].

Flow polytopes lend themselves to subdivisions via reductions, as explained below. A similar property of root polytopes was studied in $[8,9]$.

Definition 1. Given a graph $G$ on the vertex set $[n]$ containing edges $(i, j)$ and $(j, k)$, $i<j<k$, performing the reduction on these edges of $G$ yields three graphs on the vertex set $[n]$ :

$$
\begin{aligned}
& E\left(G_{1}\right)=E(G) \backslash\{(j, k)\} \cup\{(i, k)\}, \\
& E\left(G_{2}\right)=E(G) \backslash\{(i, j)\} \cup\{(i, k)\}, \\
& E\left(G_{3}\right)=E(G) \backslash\{(i, j),(j, k)\} \cup\{(i, k)\} .
\end{aligned}
$$

When performing a reduction on the edges $(i, j),(j, k)$ we say that the edge $(i, j)$ is dropped if we go towards $G_{2}$ or $G_{3}$ as in $(1)$ and $(i, j)$ is kept if we go towards $G_{1}$. Similarly, edge $(j, k)$ is dropped if we go towards $G_{1}$ or $G_{3}$ as in $(1)$ and $(j, k)$ is kept if we go towards $G_{2}$. 


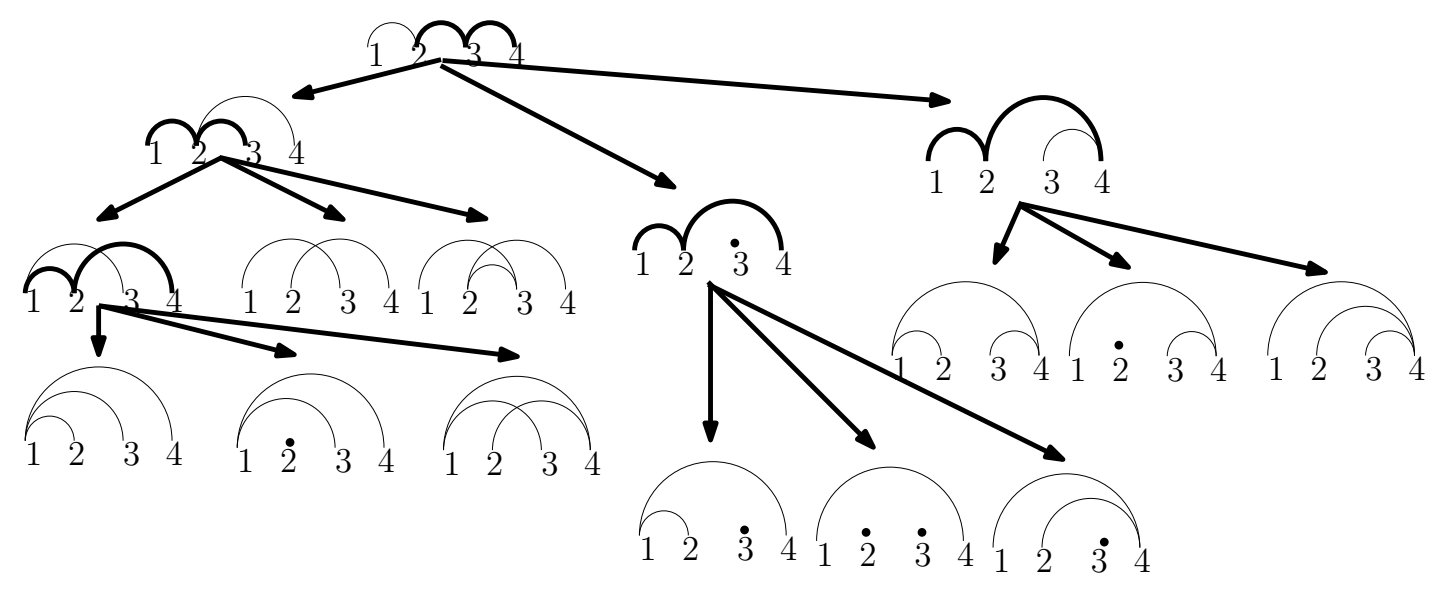

Figure 1: A reduction tree of $G=([4],\{(1,2),(2,3),(3,4)\})$. The edges on which the reductions are performed are in bold.

Definition 2. A reduction tree $R_{G}$ of a graph $G$ is a tree with nodes labeled by graphs and such that all non-leaf nodes of $R_{G}$ have three children. The root is labeled by $G$. If there are two edges $(i, j),(j, k) \in E(G), i<j<k$, on which we choose to do a reduction, then the children of the root are labeled by $G_{1}, G_{2}$ and $G_{3}$ as in (1). Next, continue this way by constructing reduction trees for $G_{1}, G_{2}$ and $G_{3}$. If some graph has no edges $(i, j),(j, k), i<j<k$, then it is its own reduction tree. Note that the reduction tree $R_{G}$ is not unique; it depends on our choice of edges to reduce. However, the number of leaves (referring to the graph labeling a leaf) of all reduction trees of $G$ with a given number of edges is the same as Lemma 4 states below. We choose a particular embedding of the reduction tree in the plane for convenience: we root it at $G$ with the tree growing downwards, and such that the left child is $G_{1}$, the middle child is $G_{3}$ and the right child is $G_{2}$; see Figure 1 . The leaves which have the same number of edges at the root are called full dimensional.

Definition 3. Let the edges of $G$ be $e_{1}, \ldots, e_{k}$, where we distinguish multiple edges. If a reduction involving edges $a=(i, j)$ and $b=(j, k)$ of $G$ is performed, then the new edge $(i, k)$ appearing in all three graphs as in (1) is formally thought of as $a+b(=b+a)$. The other edges stay unchanged. To get to nodes $G_{1}$ and $G_{2}$ of $R_{G}$ we iterate this process, thereby expressing the edges of any node as a sum of edges of the graph being the root of the reduction tree. Two edges $c$ and $d$ in the graphs $G_{1}$ and $G_{2}$, respectively, are the same, if they are the sum of exactly the same edges of $G$. The intersection of two graphs $G_{1}$ and $G_{2}$ in a reduction tree $R_{G}$ is $G_{1} \cap G_{2}=\left(V(G), E\left(G_{1}\right) \cap E\left(G_{2}\right)\right)$, where if $e \in E\left(G_{1}\right) \cap E\left(G_{2}\right)$ then as explained above $e$ is the sum of the same edges of $G$ in both $G_{1}$ and $G_{2}$. See Figure 2 for an illustration.

Lemma 4. [8] Given two distinct reduction trees of $G$, let $r_{k}^{1}$ and $r_{k}^{2}$ be the number of leaves with $k$ edges is them, respectively. Then, $r_{k}^{1}=r_{k}^{2}$. 


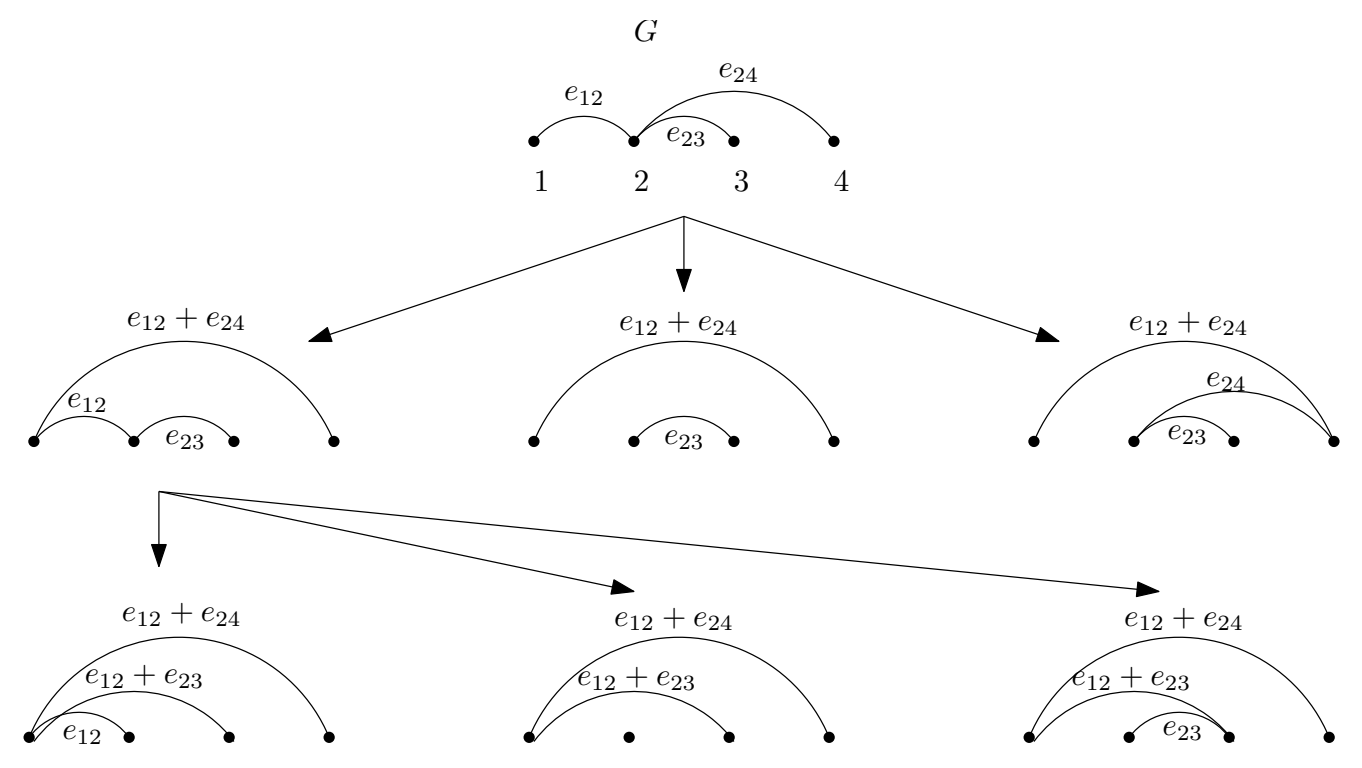

Figure 2: The edges of the nodes of a reduction tree of $G$ are considered as formal sums of the edges of $G$.

Definition 5. The augmented graph $\widetilde{G}$ of $G=([n], E)$ is $\widetilde{G}=([n] \cup\{s, t\}, \widetilde{E})$, where $s$ (source) is the smallest, $t$ (target/sink) is the biggest vertex of $[n] \cup\{s, t\}$, and $\widetilde{E}=$ $E \cup\{(s, i),(i, t) \mid i \in[n]\}$. Denote by $\mathcal{P}(\widetilde{G})$ the set of all maximal paths in $\widetilde{G}$, referred to as routes. It is well known that the unit flows sent along the routes in $\mathcal{P}(\widetilde{G})$ are the vertices of $\mathcal{F}(\widetilde{G})[15$, Section $13.1 \mathrm{a}]$.

Definition 6. Consider a node $G_{1}$ of the reduction tree $R_{G}$, where each edge of $G_{1}$ is considered as a sum of the edges of $G$. The map $m: E\left(G_{1}\right) \rightarrow \mathcal{P}(\widetilde{G})$ takes an edge $\left(v_{1}, v_{2}\right)=$ $e=e_{i_{1}}+\cdots+e_{i_{l}}, e \in G_{1}, e_{i_{j}} \in E(G), j \in[l]$, to the route $\left(s, v_{1}\right), e_{i_{1}}, \ldots, e_{i_{l}},\left(v_{2}, t\right)$. Then the vertices of $\mathcal{F}_{\widetilde{G_{1}}}$ with respect to $G$ are the unit flows along the the routes that are in the image of $m$ and the routes of the form $(s, v),(v, t)$, where $v$ is any vertex of $G$. In case $G_{1}$ is not a node of the reduction tree $R_{G}$, but it is an intersection of nodes of $R_{G}$, so that each edge of $G_{1}$ can still be considered as a sum of the edges of $G$, we still define $\mathcal{F}_{\widetilde{G_{1}}}$ as above. This definition of $\mathcal{F}_{\widetilde{G_{1}}}$ is of course with respect to $G$, and this is understood from the context. For an example see Figure 3

Using the above definitions the proof of the following lemma is an easy exercise.

Lemma 7. [11, Proposition 1],[13, Proposition 4.1], [14, 18] Given a graph $G$ on the vertex set $[n]$ and $(i, j),(j, k) \in E(G)$, for some $i<j<k$, and $G_{1}, G_{2}, G_{3}$ as in (1) and $\mathcal{F}_{\widetilde{G}_{i}}, i \in[3]$, as in Definition 6 we have

$$
\mathcal{F}_{\widetilde{G}}=\mathcal{F}_{\widetilde{G}_{1}} \bigcup \mathcal{F}_{\widetilde{G}_{2}}, \mathcal{F}_{\widetilde{G}_{1}} \bigcap \mathcal{F}_{\widetilde{G}_{2}}=\mathcal{F}_{\widetilde{G}_{3}} \text { and } \mathcal{F}_{\widetilde{G}_{1}}^{\circ} \bigcap \mathcal{F}_{\widetilde{G}_{2}}^{\circ}=\varnothing,
$$

where $\mathcal{F}_{\widetilde{G}}, \mathcal{F}_{\widetilde{G}_{1}}, \mathcal{F}_{\widetilde{G}_{2}}$ are of the same dimension $d-1, \mathcal{F}_{\widetilde{G}_{3}}$ is $d-2$ dimensional, and $\mathcal{P}^{\circ}$ denotes the interior of $\mathcal{P}$. 


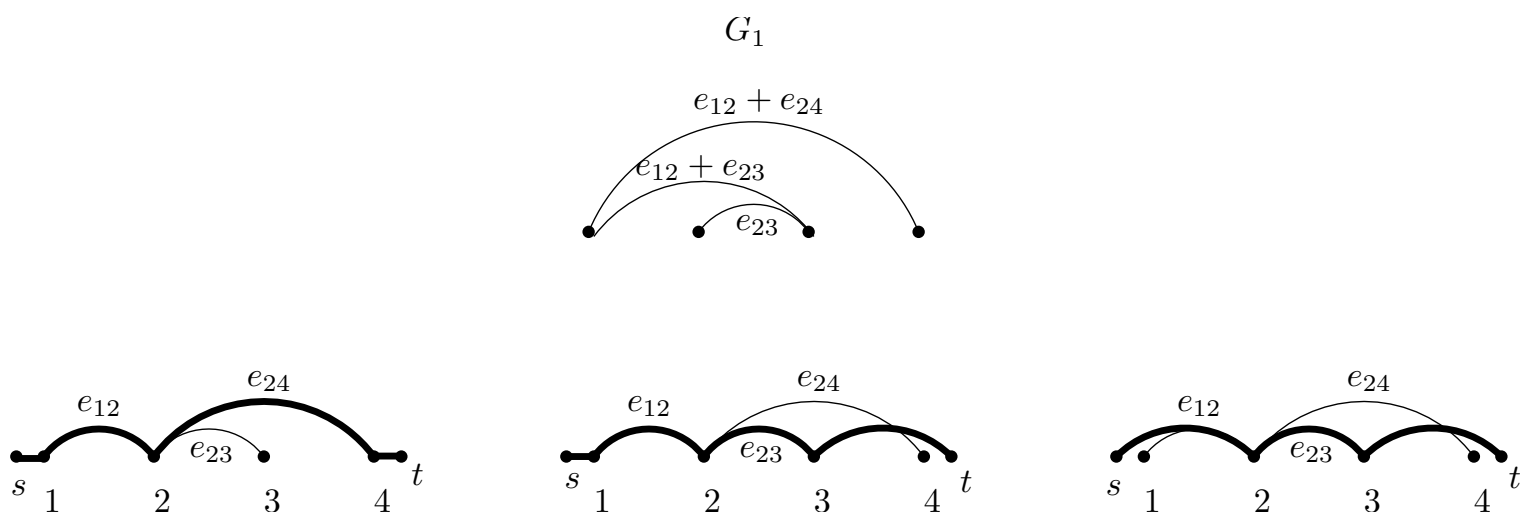

Figure 3: The graph $G_{1}$ is a node of the reduction tree give in Figure 2. The flow polytope corresponding to this node of the reduction tree is the convex hull of the points given by the unit flows on the bold routes as well as unit flows given along routes of the form $(s, v),(v, t)$, where $v \in[4]$. This flow polytope is part of a dissection of the flow polytope of the graph labeling the root, as explained in Lemma 7 .

The essence of Lemma 7 is that when we replace $G$ by the three graphs $G_{1}, G_{2}, G_{3}$ we dissected $\mathcal{F}_{\widetilde{G}}$ into polytopes $\mathcal{F}_{\widetilde{G}_{1}}$ and $\mathcal{F}_{\widetilde{G}_{2}}$ and they intersect along $\mathcal{F}_{\widetilde{G}_{3}}$. Iterating this, we obtain that the flow polytopes corresponding to the leaves of a reduction tree of $G$ as defined in Definition 6 are the interior simplices of a dissection of the flow polytope $\mathcal{F}_{\widetilde{G}}$.

\subsection{Encoding subdivisions by relations}

Note that the reduction of graphs given in (1) can be encoded as the following relation:

$$
x_{i j} x_{j k}=x_{i k} x_{i j}+x_{j k} x_{i k}+\beta x_{i k}, \text { for } 1 \leqslant i<j<k \leqslant n .
$$

Namely, interpreting the double indices of the variables $x_{i j}$ as edges, the monomial $x_{i j} x_{j k}$ picks out two edges $(i, j),(j, k), i<j<k$, and replaces it with three monomials, corresponding to operation on graphs (1). The variable $\beta$ is simply a placeholder, indicating that the number of edges in the third graph is one less than in the other graphs

These relations give rise to what we call the subdivision algebra.

Definition 8. The associative subdivision algebra, denoted by $\mathcal{S}(\beta)$, is an associative algebra, over the ring of polynomials $\mathbb{Z}[\beta]$, generated by the set of elements $\left\{x_{i j}: 1 \leqslant i<\right.$ $j \leqslant n\}$, subject to the relations:

(a) $x_{i j} x_{k l}=x_{k l} x_{i j}$, if $i<j, k<l$,

(b) $x_{i j} x_{j k}=x_{i k} x_{i j}+x_{j k} x_{i k}+\beta x_{i k}$, if $1 \leqslant i<j<k \leqslant n$.

The algebra $\mathcal{S}(\beta)$ has been studied in the context of root polytopes [8].

Definition 9. Given a monomial $M$ in $\mathcal{S}(\beta)$, its reduced form is defined as follows. Starting with $p_{0}=M$, produce a sequence of polynomials $p_{0}, p_{1}, \ldots, p_{m}$ in the following 
fashion. To obtain $p_{r+1}$ from $p_{r}$, choose a monomial of $p_{r}$ which is divisible by $x_{i j} x_{j k}$, for some $i, j, k$, and replace the factor $x_{i j} x_{j k}$ in this monomial with $x_{i k} x_{i j}+x_{j k} x_{i k}+\beta x_{i k}$. Note that $p_{r+1}$ has two more monomials than $p_{r}$. Continue this process until a polynomial $p_{m}$ is obtained, in which no monomial is divisible by $x_{i j} x_{j k}$, for any $i, j, k$. Such a polynomial $p_{m}$ is a reduced form of $M$. Note that we allow the use of the commutation relations in this process.

A possible sequence of reductions in algebra $\mathcal{S}(\beta)$ yielding a reduced form of $x_{12} x_{23} x_{34}$ is given by

$$
\begin{aligned}
x_{12} \boldsymbol{x}_{\mathbf{2 3}} \boldsymbol{x}_{\mathbf{3 4}} \rightarrow & \boldsymbol{x}_{\mathbf{1 2}} x_{24} \boldsymbol{x}_{\mathbf{2 3}}+\boldsymbol{x}_{\mathbf{1 2}} x_{34} \boldsymbol{x}_{\mathbf{2 4}}+\beta \boldsymbol{x}_{\mathbf{1 2}} \boldsymbol{x}_{\mathbf{2 4}} \\
\rightarrow & \boldsymbol{x}_{\mathbf{2 4}} x_{13} \boldsymbol{x}_{\mathbf{1 2}}+x_{24} x_{23} x_{13}+\beta x_{24} x_{13}+x_{34} x_{14} x_{12}+x_{34} x_{24} x_{14} \\
& +\beta x_{34} x_{14}+\beta x_{14} x_{12}+\beta x_{24} x_{14}+\beta^{2} x_{14} \\
\rightarrow & x_{13} x_{14} x_{12}+x_{13} x_{24} x_{14}+\beta x_{13} x_{14}+x_{24} x_{23} x_{13}+\beta x_{24} x_{13} \\
& +x_{34} x_{14} x_{12}+x_{34} x_{24} x_{14}+\beta x_{34} x_{14}+\beta x_{14} x_{12}+\beta x_{24} x_{14} \\
& +\beta^{2} x_{14}
\end{aligned}
$$

where the pair of variables on which the reductions are performed is in boldface. The reductions are performed on each monomial separately.

Given a monomial $M$ we can encode it by a graph $G_{M}$, simply by letting the edges of $G_{M}$ be the given by the indices of the variables in $M$. Denote a reduced form of $M$ in $\mathcal{S}(\beta)$ by $Q_{G_{M}}^{\mathcal{S}(\beta)}(\mathbf{x} ; \beta)$. Note that the reduced form of $M$ is not unique, so if we wanted to specify the reduced form exactly, we would also need to specify a reduction tree for $G$. When writing $Q_{G_{M}}^{\mathcal{S}(\beta)}(\mathbf{x} ; \beta)$ we pick an arbitrary reduced form at hand. If in the reduced forms we set $\mathbf{x}=(1, \ldots, 1)$, then in the notation we omit $\mathbf{x}: Q_{G_{M}}^{\mathcal{S}(\beta)}(\beta)$. We will show later that $Q_{G_{M}}^{\mathcal{S}(\beta)}(\beta)$ is independent of the choice of reduction tree, thus $Q_{G_{M}}^{\mathcal{S}(\beta)}(\beta)$ is well-defined as is.

It is easy to see that by definition, a reduced form of a monomial in the subdivision algebra can be read off from the leaves of the reduction tree of the corresponding graph. With this and Lemma 7 in mind, it is no surprise that we can prove results about reduced forms of monomials in the algebras using flow polytopes.

Recall that a reduced form of a monomial in $\mathcal{S}(\beta)$ is not necessarily unique, which could be a desirable property. Amazingly, there is a noncommutative algebra, denoted $\widetilde{A C Y B_{n}}(\beta)$, which is much like $\mathcal{S}(\beta)$, yet in which the reduced forms are unique. The latter statement was proved in [8]. It was A.N. Kirillov $[6,7]$ who introduced $\widetilde{A C Y B_{n}}(\beta)$ and shed the first light on its rich combinatorial structure. The paper [10] addresses more of the story of subdivision algebras as well as the story of $\widetilde{A C Y B_{n}}(\beta)$.

\section{Triangulating flow polytopes}

In this section we prove that we can use the subdivision algebra to obtain triangulations of every flow polytope $\mathcal{F}_{\widetilde{G}}$. A priori this is far from clear - we are only guaranteed dissections 


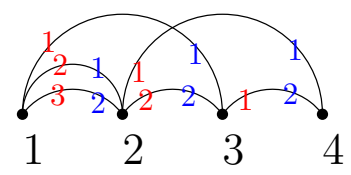

Figure 4: The ordering of the incoming and outgoing edges at each vertex from topmost to bottom.

of flow polytopes, but not the simplicial complex structure. Moreover, the triangulation we obtain is flag and regular, and thus shellable. We construct our special triangulation by picking a specific reduction order $\sigma$ on our graph $G$ and utilizing the properties of $\sigma$ to prove properties about the leaves of the reduction tree $R_{G}^{\sigma}$ obtained using $\sigma$. We then note that there is a whole class of orders for which our arguments work, but for clarity we lay out the argument for $\sigma$ first.

\subsection{A family of orders $\mathcal{F}(\sigma)$}

Given an arbitrary graph $G$ on the vertex set $[n]$, put a total order on the set of incoming and as well as a total order set of outgoing edges at each vertex $1<v<n$ (thus we consider each edge twice here, once as an incoming edge and once as an outgoing edge). Do the reductions in $G$ proceeding from the smallest vertex towards the greatest in order. Look for the smallest vertex $v$ which is nonalternating, that is that has both an edge $(a, v)$ and an edge $(v, b)$ incident to it, with $a<v<b$. (Two edges $(a, v)$ and $(v, b)$ are non-alternating if $a<v<b$.) Look at the incoming and outgoing edges at $v,(a, v)$ and $(v, b)$, which are smallest in the ordering of the incoming and outgoing edges, respectively. Do the reduction on $(a, v)$ and $(v, b)$. In the three obtained graphs the relative ordering of the edges stays the same, with the new edge $(a, b)$ either taking the place of $(a, v)$ or $(v, b)$ if these were dropped, or directly preceeding them when they are kept. Continue in this fashion on each leaf of the partial reduction tree ultimately arriving to the reduction tree $R_{G}$ with all leaves alternating graphs, that is all of their vertices are alternating. Let $\sigma$ be the order where the initial ordering of the incoming and outgoing edges at each vertex is such that the topmost is the smallest, then the next topmost, etc. See Figure 4 for an example of this ordering. All results of this paper generalize for any order in $\mathcal{F}(\sigma)$, but for simplicity we state and prove them for $\sigma$ only.

The main result of this section is:

Theorem 10. The simplices corresponding to the full dimensional leaves of $R_{G}^{\sigma}$ yield the top dimensional simplices in a regular and flag triangulation of $\mathcal{F}_{\widetilde{G}}$. Moreover, lower dimensional simplices of this triangulation which are not contained in the boundary of $\mathcal{F}_{\widetilde{G}}$ are obtained from the (not full dimensional) leaves of $R_{G}^{\sigma}$.

Proof. The proof follows from Theorem 11, Lemma 12 and Proposition 13.

Next we explain Theorem 11 and prove Proposition 13. 


\subsection{Coherent routes, cliques and triangulation of flow polytopes}

Given a graph $G$ on the vertex set $[n]$ with edges oriented from smaller to bigger vertices, the vertices of the flow polytope $\mathcal{F}_{G}$ correspond to integer flows of size one on maximal directed paths from the source (1) to the sink $(n)$. Following [3] we call such maximal paths routes. The following definitions follow [3]. Fix a framing at each inner vertex $v$ (that is a vertex that is not a source or a sink) of $G$, which is the linear ordering $\prec_{i n(v)}$ on the set of incoming edges $i n(v)$ to $v$ and the linear ordering $\prec_{\text {out }(v)}$ on the set of outgoing edges out $(v)$ from $v$. We call a graph with a framing at each inner vertex framed. For a framed graph $G$ and an inner vertex $v$ we denote by $\operatorname{In}(v)$ and by $\operatorname{Out}(v)$ the set of maximal paths ending in $v$ and the set of maximal paths starting at $v$, respectively. We define the order $\prec_{\operatorname{In}(v)}$ on the paths in $\operatorname{In}(v)$ as follows. If $P, Q \in \operatorname{In}(v)$ then let $w$ be the largest vertex after which $P$ and $Q$ coincide and before which they differ. Let $e_{P}$ be the edge of $P$ entering $w$ and $e_{Q}$ be the edge of $Q$ entering $w$. Then $P \prec_{I n(v)} Q$ if and only if $e_{P} \prec_{i n(w)} e_{Q}$. The linear order $\prec_{\text {Out }(v)}$ on $\operatorname{Out}(v)$ is defined analogously.

Given a route $P$ with an inner vertex $v$ denote by $P v$ the maximal subpath of $P$ entering $v$ and by $v P$ the maximal subpath of $P$ leaving $v$. We say that the routes $P$ and $Q$ are coherent at a vertex $v$ which is an inner vertex of both $P$ and $Q$ if the paths $P v, Q v$ are ordered the same way as $v P, v Q$; e.g., if $P v \prec_{\operatorname{In}(v)} Q v$ and $v P \prec_{\text {Out }(v)} v Q$. We say that routes $P$ and $Q$ are coherent if they are coherent at each common inner vertex. We call a set of mutually coherent routes a clique. The following theorem is a special case of $[3$, Theorems $1 \& 2]$.

Theorem 11. [3, Theorems 1 \& 2] Given a framed graph G, taking the convex hulls of the vertices corresponding to the routes in maximal cliques yield the top dimensional simplices in a regular triangulation of $\mathcal{F}_{G}$. Moreover, lower dimensional simplices of this triangulation are obtained as convex hulls of the vertices corresponding to the routes in (not maximal) cliques.

Lemma 12. The triangulation described in Theorem 11 is flag.

Proof. Consider a non-face $N$ of the triangulation that is of cardinality greater than 2 . By Theorem 11 the vertices of $N$ are routes that are not coherent; in particular there are two routes $P$ and $Q$ which yield vertices of $N$ and are not coherent. Since $P$ and $Q$ are not coherent, they constitute a non-face. Therefore, all minimal non-faces of the triangulation described in Theorem 11 are of size 2, and therefore the triangulation is flag.

Now we are ready to prove the proposition which together with Theorem 11 and Lemma 12 implies Theorem 10. We define the framing $\widetilde{\sigma}$ on $\widetilde{G}$ as the ordering $\sigma$ on the edges of $G$, that is, the incoming edges are ordered top to bottom and the outgoing edges are also ordered top to bottom, and the edges of the form $(s, i)$ and $(i, t)$, for $i \in[n]$, are always last in the orderings. See Figure 5 for an example.

Proposition 13. The set of vertices of the simplices corresponding to the leaves of $R_{G}^{\sigma}$ form a clique of mutually coherent paths in $\widetilde{G}$ with the framing $\widetilde{\sigma}$. 


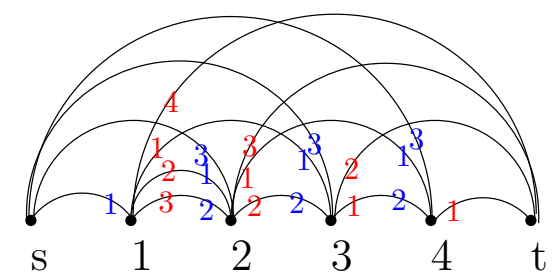

Figure 5: Framing $\widetilde{\sigma}$ on $\widetilde{G}$ with $G=([4],\{\{(1,2),(1,2),(1,3),(2,3),(2,4),(3,4)\}\})$.

Proof. Suppose that to the contrary, there are two vertices of a simplex corresponding to a leaf of $R_{G}^{\sigma}$, which correspond to non-coherent routes $P$ and $Q$ in $\widetilde{G}$. Suppose that $P$ and $Q$ are not coherent at the common inner vertex $v$. Suppose that the smallest vertex after which $P v$ and $Q v$ agree is $w_{1}$ and the largest vertex before which $v P$ and $v Q$ agree is $w_{2}$. Let the edges incoming to $w_{1}$ be $e_{P}^{1}$ and $e_{Q}^{1}$ for $P$ and $Q$, respectively, and let the edges outgoing from $w_{2}$ be $e_{P}^{2}$ and $e_{Q}^{2}$ for $P$ and $Q$, respectively. Since $P$ and $Q$ are not coherent at $v$, this implies that either $e_{P}^{1} \prec_{\text {in }\left(w_{1}\right)} e_{Q}^{1}$ and $e_{Q}^{2} \prec_{\text {out }\left(w_{2}\right)} e_{P}^{2}$ or $e_{Q}^{1} \prec_{\text {in }\left(w_{1}\right)} e_{P}^{1}$ and $e_{P}^{2} \prec_{\text {out }\left(w_{2}\right)} e_{Q}^{2}$. We also have that the segments of $P$ and $Q$ between $w_{1}$ and $w_{2}$ coincide. Note that since the edges of the form $(s, i)$ and $(i, t), i \in[n]$, are last in the linear orderings of the incoming and outgoing edges, it follows that at most one of the edges $e_{P}^{1}$ and $e_{P}^{2}$ and at most one of the edges $e_{Q}^{1}$ and $e_{Q}^{2}$ could be incident to $s$ or $t$. We consider several cases based on whether any of $e_{P}^{1}, e_{P}^{2}, e_{Q}^{1}, e_{Q}^{2}$ are incident to $s$ or $t$. Denote by $p$ the sum of edges between $w_{1}$ and $w_{2}$ on $P$. If none of $e_{P}^{1}, e_{P}^{2}, e_{Q}^{1}, e_{Q}^{2}$ are incident to $s$ or $t$, then after a certain number of reductions executed according to $\sigma$ we are about the perform the reduction $\left(*\left(e_{\bar{Z}}^{1}+p\right), e_{Z}^{2}\right)$, where $*\left(e_{\bar{Z}}^{1}+p\right)$ denotes the sum of edges left of $w_{1}$ that are edges in $\bar{Z}$ not incident to $s$ (including $e_{\bar{Z}}^{1}$ in particular) and $p,\{\bar{Z}, Z\}=\{P, Q\}$. Note, however, that after executing this reduction we have to drop either the edge $*\left(e_{\bar{Z}}^{1}+p\right)$ or the edge $e_{Z}^{2}$. However, if the former were true, it would make it impossible for $*\left(e_{\bar{Z}}^{1}+p\right)+e_{\bar{Z}}^{2}$ to be a subsum in an edge of the leaf we are considering, which it has to be in order for $\bar{Z}$ to be a route giving a vertex of the simplex we are considering. The latter on the other hand would make it impossible for $*\left(e_{Z}^{1}+p\right)+e_{Z}^{2}$ to be a subsum in an edge of the leaf we are considering, where $*\left(e_{Z}^{1}+p\right)$ denotes the sum of edges left of $w_{1}$ that are edges in $Z$ not incident to $s$. However then $Z$ cannot be a route giving a vertex of the simplex we are considering. Thus we see that $Z$ and $\bar{Z}$, aka, $P$ and $Q$, cannot be incoherent in this way. It follows that we need to consider the possibilities where some of $e_{P}^{1}, e_{P}^{2}, e_{Q}^{1}, e_{Q}^{2}$ are incident to $s$ or $t$. One can construct similar arguments to the above in all those cases.

\section{Reduced forms are $h$-polynomials}

In this section we show that for a graph $G$ the reduced form of the monomial $m[G]=$ $\prod_{(i, j) \in E(G)} x_{i j}$ can be seen as the shifted $h$-polynomial of a unimodular triangulation of the flow polytope $\mathcal{F}_{\widetilde{G}}$. 
Definition 14. Let $\mathcal{C}$ be a $d-1$ dimensional simplicial complex. The $\boldsymbol{f}$-vector of $\mathcal{C}$ is

$$
f(\mathcal{C})=\left(f_{-1}, f_{0}, \ldots, f_{d-1}\right)
$$

where $f_{i}=f_{i}(\mathcal{C})$ be the number of $i$-dimensional simplices in $\mathcal{C}$. By convention, $f_{-1}=1$ unless $\mathcal{C}=\varnothing$, in which case $f_{-1}=0$. The $\boldsymbol{h}$-vector of $\mathcal{C}$ is $h(\mathcal{C})=\left(h_{0}, h_{1}, \ldots, h_{d}\right)$, defined by

$$
\sum_{i=0}^{d} f_{i-1}(x-1)^{d-i}=\sum_{i=0}^{d} h_{i} x^{d-i}
$$

Define the $\boldsymbol{h}$-polynomial of a simplicial complex $\mathcal{C}$ to be

$$
h(\mathcal{C}, \mathbf{x})=\sum_{i=0}^{d} h_{i} x^{i}
$$

Lemma 15. [16] For a simplicial complex $\mathcal{C}$ we have:

$$
h(\mathcal{C}, \mathbf{x})=\sum_{F \in \mathcal{C}} x^{\# F}(1-x)^{d-\# F} .
$$

The main result of this section is the following theorem.

Theorem 16. We have

$$
Q_{G}^{\mathcal{S}(\beta)}(\beta)=h(\mathcal{C}, \beta+1),
$$

where $\mathcal{C}$ is any unimodular triangulation of $\mathcal{F}_{\widetilde{G}}$.

We illustrate the theorem on an example in Figure 6.

To prove Theorem 16, we use the following lemma, which is implicit in [16]. For completeness, we include a proof of it.

Lemma 17. Let $\mathcal{C}$ be a $(d-1)$-dimensional simplicial complex homeomorphic to a ball and $f_{i}^{\circ}$ be the number of interior faces of $\mathcal{C}$ of dimension $i$. Then

$$
h(\mathcal{C}, \beta+1)=\sum_{i=0}^{d-1} f_{i}^{\circ} \beta^{d-1-i}
$$

Proof. We have that

$$
h(\mathcal{C}, \beta+1)=\sum_{F \in \mathcal{C}}(\beta+1)^{\# F}(-\beta)^{d-\# F}=\sum_{i=-1}^{d-1} f_{i}(\beta+1)^{i}(-\beta)^{d-i-1} .
$$

Thus, (8) is equivalent to

$$
\sum_{i=-1}^{d-1} f_{i}(\beta+1)^{i}(-\beta)^{d-i-1}=\sum_{i=0}^{d-1} f_{i}^{\circ} \beta^{d-1-i}
$$




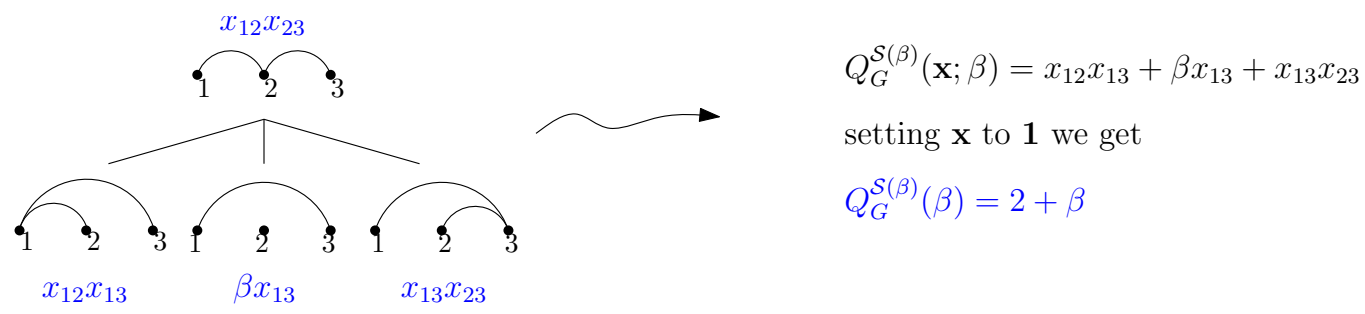

The triangulation $\mathcal{C}$ corresponding

to the above reduction tree consists of two

$h(\mathcal{C}, \beta+1)=2+\beta$

4-dimensional simplices $\Delta_{1}$ and $\Delta_{2}$ and

their faces; $\Delta_{1}$ and $\Delta_{2}$ attach to each

other on a 3 -dimensional face.

Figure 6: On the left is a reduction tree of $G=([2],\{(1,2),(2,3)\})$ with the monomials corresponding to the graphs noted and a description of the corresponding triangulation $\mathcal{C}$. On the right is the reduced form corresponding to the reduction tree and the (shifted) $h$-polynomial of $\mathcal{C}$. As in Theorem 16 we get $Q_{G}^{\mathcal{S}(\beta)}(\beta)=h(\mathcal{C}, \beta+1)$.

which is what we proceed to prove now. For brevity, denote the left hand side of (9) by $L H S$ and the right hand side by $R H S$. Then we see that

$$
\left[\beta^{d}\right] L H S=(-1)^{d}+\sum_{i=0}^{d-1} f_{i}(-1)^{d-i-1}\left(\begin{array}{c}
i+1 \\
0
\end{array}\right) .
$$

For $j \in[d]$ we have that

$$
\left[\beta^{d-j}\right] L H S=\sum_{i=j-1}^{d-1} f_{i}(-1)^{d-i-1}\left(\begin{array}{c}
i+1 \\
j
\end{array}\right) .
$$

On the other hand, $\left[\beta^{d}\right] R H S=0$ and for $j \in[d]$ we have $\left[\beta^{d-j}\right] R H S=f_{j-1}^{\circ}$. Thus, (9) is equivalent to

$$
(-1)^{d}+\sum_{i=0}^{d-1} f_{i}(-1)^{d-i-1}\left(\begin{array}{c}
i+1 \\
0
\end{array}\right)=0
$$

and for $j \in[d]$

$$
\sum_{i=j-1}^{d-1} f_{i}(-1)^{d-i-1}\left(\begin{array}{c}
i+1 \\
j
\end{array}\right)=f_{j-1}^{\circ} .
$$

Note that (10) is the familiar Euler characteristic expression of a simplicial complex that is homeomorphic to a ball. To prove (11) we interpret its left hand side as an inclusion exclusion formula for $f_{j-1}^{\circ}$. Indeed, $f_{d-1}\left(\begin{array}{c}d \\ j\end{array}\right)$ is the number of $(j-1)$-faces of the $f_{d-1}$ top dimensional simplices in $\mathcal{C}$, with overcounting. To correct for the overcounting 
we substract $f_{d-2}\left(\begin{array}{c}d-1 \\ j\end{array}\right)$, which is the number of $(j-1)$-faces of the $f_{d-2}(d-2)$-simplices of $\mathcal{C}$. Note that here we therefore also take out all $(j-1)$-faces that are on the boundary of $\mathcal{C}$. Again, we have oversubstracted, so we add back $f_{d-3}\left(\begin{array}{c}d-2 \\ j\end{array}\right)$, etc., to arrive to $f_{j-1}^{\circ}$. We used that $\mathcal{C}$ is a pure simplicial complex when we assumed that all $(j-1)$-faces are a face of a $(d-1)$-face.

Proof of Theorem 16. Note that, by definition, the reduced form $Q_{G}^{\mathcal{S}(\beta)}(\beta)$ read off from the reduction tree $R_{G}^{\sigma}$, which yields the unimodular triangulation $\mathcal{C}^{\sigma}$, can be written as

$$
Q_{G}^{\mathcal{S}(\beta)}(\beta)=\sum_{i=1}^{d-1} f_{i}^{\circ} \beta^{d-1-i},
$$

where $\mathcal{C}^{\sigma}$ is $d-1$ dimensional and $f_{i}^{\circ}$ is the number of leaves of $R_{G}^{\sigma}$ yielding an $i$-dimensional simplex. The notation $f_{i}^{\circ}$ signifies that the union of the open simplices corresponding to the leaves of a reduction tree yield the open polytope $\mathcal{F}_{\widetilde{G}}$. By Lemma 17 we have that

$$
\sum_{i=1}^{d-1} f_{i}^{\circ} \beta^{d-1-i}=h\left(\mathcal{C}^{\sigma}, \beta+1\right) .
$$

Since by Lemma 4 we know that $Q_{G}^{\mathcal{S}(\beta)}(\beta)$ does not depend on the particular reduction tree, we are done.

\section{A description of the leaves of $\boldsymbol{R}_{G}^{\sigma}$}

In this section we describe all the leaves of the reduction tree $R_{G}^{\sigma}$ in terms of shellings of the triangulation obtained from $R_{G}^{\sigma}$ as in Theorem 10. Since we know that these triangulations are regular, it follows that they are also shellable.

Theorem 18. Let $\mathcal{F}_{\widetilde{F}_{1}}, \ldots, \mathcal{F}_{\widetilde{F}_{l}}$ be a shelling order of the simplicial complex arising from $R_{G}^{\sigma}$. Let

$$
P_{i}:=\left\{\left\{Q_{1}^{i}, \ldots, Q_{f(i)}^{i}\right\}\right\}=\left\{\left\{F_{i} \cap F_{j}|1 \leqslant j<i,| E\left(F_{i} \cap F_{j}\right)|=| E\left(F_{i}\right) \mid-1\right\}\right\} .
$$

Then

$$
\sum_{i=1}^{l} \prod_{j=1}^{f(i)}\left(F_{i}+Q_{j}^{i}\right)
$$

is the formal sum of the set of the leaves of $R_{G}^{\sigma}$, where the product of graphs is their intersection. If $f(i)=0$ we define $\prod_{j=1}^{f(i)}\left(F_{i}+Q_{j}^{i}\right)=F_{i}$.

Before proving Theorem 18, we record a few properties of flow polytopes that easily follow from the above considerations and from the fact that the dimension of $\mathcal{F}_{G}$ is $|E(G)|-|V(G)|+1[1]$. In both lemmas the meanings of $\mathcal{F}_{\widetilde{H}}$ for a node $H$ of $R_{G}^{\sigma}$ or intersection of such nodes is as in Definition 6, which is the key to the proofs that are left to the interested reader. 
Lemma 19. Let $G_{1}$ and $G_{2}$ be two leaves of $R_{G}^{\sigma}$. Then

$$
\mathcal{F}_{\widetilde{G_{1}}} \cap \mathcal{F}_{\widetilde{G_{2}}}=\mathcal{F}_{\widetilde{G_{1} \cap G_{2}}} \cdot
$$

Moreover, $G_{1} \cap G_{2}$ is a leaf of $R_{G}^{\sigma}$ if and only if $\mathcal{F}_{\widetilde{G_{1}}} \cap \mathcal{F}_{\widetilde{G_{2}}}$ is not contained in the boundary of $\mathcal{F}_{\widetilde{G}}$.

Lemma 20. Let $G_{1}$ and $G_{2}$ be two leaves of $R_{G}^{\sigma}$. The dimension of $\mathcal{F}_{G_{1} \cap G_{2}}$ is $\mid E\left(G_{1} \cap\right.$ $\left.G_{2}\right)|+| V\left(G_{1} \cap G_{2}\right) \mid-1$.

Proof of Theorem 18. By Lemmas 19 and 20 we see that if $\mathcal{F}_{\widetilde{F_{1}}}, \ldots, \mathcal{F}_{\widetilde{F}_{1}}$ is a shelling order, then the set of facets on which $\mathcal{F}_{\widetilde{F}_{i}}$ attaches to $\mathcal{F}_{\widetilde{F_{1}}}, \ldots, \mathcal{F}_{\widetilde{F_{i-1}}}$ is $\left\{\mathcal{F}_{\widetilde{Q}} \mid Q \in P_{i}\right\}$. Moreover, since the intersection of two top dimensional simplices of a triangulation of $\mathcal{F}_{\widetilde{G}}$ is not contained in the boundary of $\mathcal{F}_{\widetilde{G}}$, it follows that every element of $P_{i}, i \in[l]$, appears in $R_{G}^{\sigma}$ by the second part of Lemma 19 (and it is a leaf since it is the intersection of two alternating graphs, so it is alternating itself). Using this same argument repeatedly and the fact that we can built up the polytope piece by piece by following the shelling, we obtain Theorem 18.

\section{Nonnegativity results about reduced forms}

This section is devoted to two nonnegativity results, which are consequences of the above considerations.

Theorem 21. The polynomial $Q_{G}^{\mathcal{S}(\beta)}(\beta-1)$ is a polynomial in $\beta$ with nonnegative coefficients.

Proof. Recall that by Theorem 16 we have that

$$
Q_{G}^{\mathcal{S}(\beta)}(\beta-1)=h(\mathcal{C}, \beta),
$$

where $\mathcal{C}$ is a unimodular triangulation of $\mathcal{F}_{\widetilde{G}}$. Let $\mathcal{C}$ be the abstract simplicial complex obtained from $R_{G}^{\sigma}$, as in Theorem 10. Since by Theorem 10 this triangulation is regular, and therefore it is shellable, we get that $h_{i}$ is equal to the number of top dimensional simplices which attach on $i$ facets to the union of previous simplices in a shelling order.

A nice example of the polynomial $h(\mathcal{C}, \beta)$ is in the case when we let $\mathcal{C}$ be a unimodular triangulation of $\mathcal{F}_{\widetilde{P_{n}}}$, where $P_{n}=([n],\{(i, i+1) \mid i \in[n-1]\})$. In this case the coefficients of $h(\mathcal{C}, \beta)$ are given by the Narayana numbers, which can be seen via the results $[12$, Theorem 4.4] and [17, Exercise 6.31b].

Using Theorem 21 we are ready to prove a special case of Kirillov's [6, Conjecture 2].

Conjecture 22. [6, Conjecture 2] Let $k_{1}, \ldots, k_{n-1}$ be a sequence of nonnegative integers and let $M=x_{12}^{k_{1}} x_{23}^{k_{2}} \cdots x_{n-1, n}^{k_{n-1}}$. Then the reduced form of $M$ evaluated at $\mathbf{x}=(1, \ldots, 1)$ and $\beta-1$ in $\widehat{A C Y B_{n}}(\beta)$ is a polynomial in $\beta$ with nonnegative coefficients. 
Theorem 23. [6, cf. Conjecture 2]. The reduced form of $x_{12} x_{23} \cdots x_{n-1, n}$ evaluated at $\mathbf{x}=(1, \ldots, 1)$ and $\beta-1$ in $\widetilde{A C Y B_{n}}(\beta)$ is a polynomial in $\beta$ with nonnegative coefficients.

Proof. In [8] it is proved that the monomial $x_{12} x_{23} \cdots x_{n-1, n}$ can be reduced in $\widetilde{A C Y B_{n}}(\beta)$ so that the resulting monomials in the reduced form have no variables of the form $x_{i j} x_{j k}$, $i<j<k$. Thus, reduced form of $x_{12} x_{23} \cdots x_{n-1, n}$ evaluated at $\mathbf{x}=(1, \ldots, 1)$ and $\beta-1$ in $\widetilde{A C Y B_{n}}(\beta)$ is equal to the reduced form of $x_{12} x_{23} \cdots x_{n-1, n}$ evaluated at $\mathbf{x}=(1, \ldots, 1)$ and $\beta-1$ in $\mathcal{S}(\beta)$. Therefore we can apply Theorem 21 to obtain this result.

\section{Reduced forms and Ehrhart series}

In this section we connect the $h$-polynomials of triangulations of flow polytopes to the Ehrhart series of flow polytopes, and using Theorem 16 we tie this in with reduced forms in the subdivision algebra. As a corollary to our results, we generalize [6, Theorem 3.10].

Recall that for a polytope $\mathcal{P} \subset \mathbb{R}^{N}$, the $t^{t h}$ dilate of $\mathcal{P}$ is $t \mathcal{P}=\left\{\left(t x_{1}, \ldots, t x_{N}\right) \mid\right.$ $\left.\left(x_{1}, \ldots, x_{N}\right) \in \mathcal{P}\right\}$. The number of lattice points of $t \mathcal{P}$, where $t$ is a nonnegative integer and $\mathcal{P}$ is a convex polytope, is given by the Ehrhart function $i(\mathcal{P}, t)$. If $\mathcal{P}$ has integral vertices then $i(\mathcal{P}, t)$ is a polynomial.

The following is a well-known result specialized to $\mathcal{F}_{\widetilde{G}}$.

Lemma 24. Let $\mathcal{C}$ be a unimodular triangulation of $\mathcal{F}_{\widetilde{G}}$. Then

$$
h(\mathcal{C}, \beta)=\sum_{m \geqslant 0}\left(i\left(\mathcal{F}_{\widetilde{G}}, m\right) \beta^{m}\right)(1-\beta)^{\operatorname{dim}\left(\mathcal{F}_{\widetilde{G}}\right)+1} .
$$

Corollary 25. We have

$$
Q_{G}^{\mathcal{S}(\beta)}(\beta-1)=\sum_{m \geqslant 0}\left(i\left(\mathcal{F}_{\widetilde{G}}, m\right) \beta^{m}\right)(1-\beta)^{\operatorname{dim}\left(\mathcal{F}_{\widetilde{G}}\right)+1} .
$$

Proof. Follows directly from Theorem 16 and Lemma 24.

Corollary 26. [6, Theorem 3.10]

$$
Q_{K_{n}}^{\mathcal{S}(\beta)}(\beta-1)=\sum_{m \geqslant 0}\left(i\left(C R Y_{n+1}, m\right) \beta^{m}\right)(1-\beta)^{\left(\begin{array}{c}
n+1 \\
2
\end{array}\right)} .
$$

Proof. The result follows from Corollary 25 for $G=K_{n}$, $\operatorname{since} \operatorname{dim}\left(\mathcal{F}_{\widetilde{K_{n}}}\right)=\left(\begin{array}{c}n+1 \\ 2\end{array}\right)-1$ and $i\left(C R Y_{n+1}, m\right)=i\left(\mathcal{F}_{\overparen{K_{n}}}, m\right)$, as explained in [11].

To state our final result, also a corollary of Corollary 25, relating the reduced forms to Kostant partition functions, we remind the reader of the following definition.

The Kostant partition function $K_{G}$ evaluated at the vector $\mathrm{v} \in \mathbb{Z}^{n+1}$ is defined as

$$
K_{G}(\mathrm{v})=\#\left\{\left(b_{k}\right)_{k \in[N]} \mid \sum_{k \in[N]} b_{k} \alpha_{k}=\mathrm{v} \text { and } b_{k} \in \mathbb{Z}_{\geqslant 0}\right\}
$$


where $[N]=\{1,2, \ldots, N\}$ and $\left\{\left\{\alpha_{1}, \ldots, \alpha_{N}\right\}\right\}$ is the multiset of vectors corresponding to the multiset of edges of $G$ under the correspondence which associates an edge $(i, j)$, $i<j$, of $G$ with a positive type $A_{n}$ root $e_{i}-e_{j}$, where $e_{i}$ is the $i$ th standard basis vector in $\mathbb{R}^{n+1}$. In other words, $K_{G}(\mathrm{v})$ is the number of ways to write the vector v as a $\mathbb{N}$-linear combination of the positive type $A_{n}$ roots (with possible multiplicities) corresponding to the edges of $G$, without regard to order.

Corollary 27. For any graph $G$ we have

$$
Q_{G}^{\mathcal{S}(\beta)}(\beta-1)=\sum_{m \geqslant 0}\left(K_{\widetilde{G}}(m, 0, \ldots, 0,-m) \beta^{m}\right)(1-\beta)^{\# E(G)+\# V(G)},
$$

where $K_{\widetilde{G}}$ is the Kostant partition function for $\widetilde{G}$.

Proof. Follows from Corollary 25 since $i\left(\mathcal{F}_{\widetilde{G}}, m\right)=K_{\widetilde{G}}(m, 0, \ldots, 0,-m)$ is a simple corollary of the definitions of these objects and $\operatorname{dim}\left(\mathcal{F}_{\widetilde{G}}\right)=\# E(\widetilde{G})-\# V(\widetilde{G})+1=\# E(G)+$ $\# V(G)-1$. For detailed explanations of both of these and related results see [13].

\section{Acknowledgements}

The author thanks Federico Ardila, Louis Billera and Ed Swartz for several interesting discussions regarding this work. The author also thanks Alejandro H. Morales for many inspiring conversations about flow polytopes over the course of the years and the anonymous referee for their suggestions.

\section{References}

[1] Welleda Baldoni and Michelle Vergne. Kostant partitions functions and flow polytopes. Transform. Groups, no 3-4, 447-469, 2008.

[2] Clara S. Chan, David P. Robbins and David S. Yuen. On the volume of a certain polytope. Experiment. Math., no. 1, 91-99, 2000.

[3] Vladimir I. Danilov, Alexander V. Karzanov and Gleb A. Koshevoy. Coherent fans in the space of flows in framed graphs. DMTSC proc., FPSAC 2012 Nagoya, Japan, 483-494, 2012.

[4] Sergey Fomin and Anatol N. Kirillov. Quadratic algebras, Dunkl elements, and Schubert calculus. Advances in Geometry, no. 172, 147-182, 1999.

[5] Anatol N. Kirillov. Ubiquity of Kostka polynomials. arXiv:math/9912094, 1999.

[6] Anatol N. Kirillov. On some combinatorial and algebraic properties of Dunkl elements. RIMS preprint, 2012.

[7] Anatol N. Kirillov. On some quadratic algebras, Dunkl elements, Schubert, Grothendieck, Tutte and reduced polynomials. RIMS preprint, 2014.

[8] Karola Mészáros. Root polytopes, triangulations, and the subdivision algebra, I. Trans. Amer. Math. Soc., vol. 363, no. 8, 4359-4382, 2011. 
[9] Karola Mészáros. Root polytopes, triangulations, and the subdivision algebra, II. Trans. Amer. Math. Soc., vol. 363, no. 11, 6111-6141, 2011.

[10] Karola Mészáros. h-polynomials of reduction trees. http://arxiv.org/abs/1407. 2684, 2014.

[11] Karola Mészáros. Product formulas for volumes of flow polytopes. Proc. Amer. Math. Soc., vol. 143, no. 3, 937-954, 2015.

[12] Karola Mészáros. Pipe dream complexes and triangulations of root polytopes belong together. arXiv: 1502.03991, 2015

[13] Karola Mészáros and Alejandro H. Morales. Flow polytopes of signed graphs and the Kostant partition function. Int. Math. Res. Notices no. 3: 830-871, 2015.

[14] Alexander Postnikov. Personal communication, 2010.

[15] Alexander Schrijver. Combinatorial Optimization, Volume C. Springer-Verlag Berlin Heidelberg, 2003.

[16] Richard P. Stanley. Combinatorics and Commutative Algebra, Birkhäuser, 1996.

[17] Richard P. Stanley. Enumerative combinatorics. Vol. 2, Cambridge Studies in Advanced Mathematics, no. 62, Cambridge University Press, Cambridge, 1999.

[18] Richard P. Stanley. Acyclic flow polytopes and Kostant's partition function. Conference transparencies, http://math.mit.edu/ rstan/trans.html, 2000.

[19] Doron Zeilberger. Proof of a conjecture of Chan, Robbins, and Yuen. Electron. Trans. Numer. Anal., 147-148, 1999. 